\title{
11
}

\section{Schlangenrätsel 2}

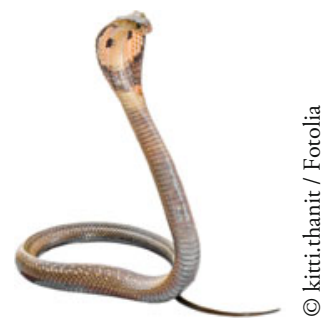

Im folgenden Buchstabenfeld hat sich wieder ein weiser Spruch eingeschlängelt. Der Anfangsbuchstabe (A), der Endbuchstabe (M), sowie zwei Zwischenbuchstaben (F, L) sind grau markiert. Umlaute werden umgewandelt zu UE, AE und OE. Findest du die Wortschlange?

\begin{tabular}{|ccccccccccccc|}
\hline E & F & G & M & U & C & S & W & B & G & H & J & K \\
Q & A & U & C & H & L & O & G & D & A & V & O & L \\
N & F & B & S & E & F & G & F & G & H & T & M & K \\
A & Y & E & V & I & D & M & E & U & L & M & O & I \\
C & H & N & R & N & A & G & Q & X & I & V & A & S \\
U & A & T & A & I & F & U & L & G & O & F & A & V \\
T & S & M & E & D & F & M & S & T & B & A & U & M \\
W & X & O & A & F & E & L & R & N & M & T & H & Y \\
H & W & R & E & W & R & K & E & D & O & H & G & T \\
K & K & M & L & L & T & M & A & L & V & N & F & N \\
I & O & L & Y & G & S & A & G & T & X & U & M & D \\
\hline
\end{tabular}

Lösung:

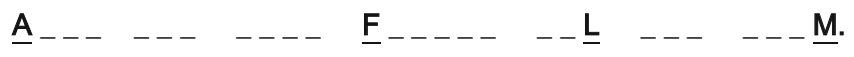

(C) Springer-Verlag Berlin Heidelberg 2016

C. Reinbold, Fetthenne, Moderlieschen, Warzenbeißer,

DOI 10.1007/978-3-662-52817-4_11 